\section{WRESTLING INJURIES DURING THE TOKYO 2020 OLYMPIC GAMES}

1,3Babak Shadgan, 'Molnar Szabolcs, 'Abaeva Elena, ${ }^{2}$ Masujima Atsushi. 'UWW Medical, Prevention and Anti-Doping Commission, Corsier-sur-Vevey, Switzerland; ${ }^{2}$ Teikyo Heisei University, Tokyo, Japan; ${ }^{3}$ University of British Columbia, Department of Orthopaedics, Vancouver, Canada

\subsection{6/bjsports-2021-IOC.207}

Background Understanding the incidence and characteristics of injuries in each sport helps to implement more effective preventive measures. Studying this information after the occurrence of the COVID-19 pandemic was particularly important. Accordingly, the medical commission of the United World Wrestling (UWW) has implemented a systematic surveillance of injuries during the Tokyo 2020 Olympic Games.

Objective To assess the injury profile of elite senior wrestlers in Men's Greco-Roman (GR), Men's Freestyle (FS), and Women's wrestling (WW) during the Tokyo 2020 Olympic Games.

Design Descriptive epidemiologic study.

Setting Clinical.

Participants 286 senior qualified wrestlers participated in Tokyo 2020 Olympic Games.

Interventions UWW injury surveillance database system.

Main Outcome Measurements Athlete's demographic, weight category, injury type, severity, location and mechanism.

Results A total of 286 athletes sustained 28 injuries during 322 matches; 9.8 injuries per 100 athletes $(12.1 \%$ in men; $5.2 \%$ in women) and 8.7 injuries per 100 bouts. Among the 3 styles, WW had the lowest injury rate $(5.2 \%)$ and FS showed the highest rate $(12.8 \%)$. More injuries were observed in the low-weight categories (64.3\%). The most common injury type was skin laceration and contusion (60.6\%) due to direct contact and the most common site of injury was head and face $(71.4 \%)$. In sum, $78.6 \%$ of all injuries were categorized as mild, $10.7 \%$ as moderate and $10.7 \%$ as severe.

Conclusions No serious or catastrophic injury was recorded during wrestling competitions of the Tokyo Olympic Games, and most injuries were minor. The overall rate of wrestling injuries during the Tokyo 2020 Olympic Games was higher than the 2016 Rio Games but lower than the 2012 London Olympic Games. The severity of injuries, however, was the lowest since the 2004 Athens Olympic Games. COVID-19 pandemic did not result in a higher rate of severe injuries, nor more overuse injuries.

\section{INJURIES IN YOUTH VOLLEYBALL PLAYERS AT A NATIONAL CHAMPIONSHIP COMPETITION: INCIDENCE, RISK FACTORS AND MECHANISM OF INJURY}

${ }^{1}$ Kenzie Vaandering, ${ }^{1}$ Derek Meeuwisse, ${ }^{2}$ Kerry J MacDonald, $1,3,4$ Paul H Eliason, ${ }^{1}$ Robert F Graham, ${ }^{1}$ Michaela K Chadder, ${ }^{10}$ Constance M Lebrun, $1,3,4,5,6,7$ Carolyn A Emery, $1,3,4,8,9$ Kathryn I Schneider. 'Sport Injury Research Prevention Centre, Faculty of Kinesiology, University of Calgary, Calgary, AB, Canada; ${ }^{2}$ volleyball Canada, Vancouver, BC, Canada; ${ }^{3}$ Alberta Children's Hospital Research Institute, University of Calgary, Calgary, AB, Canada; ${ }^{4}$ Hotchkiss Brain Institute, University of Calgary, Calgary, $A B$, Canada; ${ }^{5} O$ 'Brien Institute for Public Health, University of Calgary, Calgary, AB, Canada; ${ }^{6}$ Pediatrics, Cumming School of Medicine, University of Calgary, Calgary, $A B$, Canada; ${ }^{7}$ Community Health Sciences, Department of Pediatrics, Cumming School of Medicine, Calgary, AB, Canada; ${ }^{8}$ Sport Medicine Centre, Faculty of Kinesiology, University of Calgary, Calgary, AB, Canada; ${ }^{9}$ Evidence Sport and Spine, Calgary, $A B$, Canada; ${ }^{10}$ Glen Sather Sports Medicine Clinic, University of Alberta, Edmonton, $A B$, Canada

10.1136/bjsports-2021-IOC.208
Background Sport-related injuries present a substantial burden in youth sport. Injury surveillance data in youth volleyball is scarce. Understanding injury and concussion burden can inform prevention strategies.

Objective To evaluate injury incidence rates, types, mechanism, and potential risk factors in youth volleyball.

Design Prospective cohort study.

Setting 2018 Canadian Youth National Volleyball Tournament. Participants All tournament players were invited to participate (9616 players). 1876 players [466 males, 1391 females, mean age 16.2 years (1.26)] consented to participate (19.5\%).

Assessment of Risk Factors Sex (male/female), age group (U14, U16, U18), level of play [elite (top 30\%) vs. non-elite].

Main Outcome Measures Players completed a questionnaire (demographic information, injury, and concussion history). All medical attention injuries were recorded by tournament medical personnel via injury report form (e.g., mechanism, type). Injury was defined as any physical complaint seeking onsite medical attention. Concussion was defined using the $5^{\text {th }}$ International Consensus Conference on Concussion in Sport. Exploratory multivariable Poisson regression was used to analyze potential risk factors (sex, age group, level of play) for injury, adjusted for cluster by team and offset by athlete-exposures (AEs).

Results Of the 105 total injuries [6.15 injuries/1000 AEs (95\% CI: 5.01 to 7.47$)$, concussion was the most common $(n=28$; $26.2 \%)$, followed by knee $(n=16 ; 15.0 \%)$ and ankle injuries $(n=15 ; 14.0 \%)$. Most concussions occurred due to ball-tohead contact $(61.5 \%)$ and were unanticipated $(84.6 \%)$. There was no difference in injury risk by sex $\left(\operatorname{IRR}_{\mathrm{F} / \mathrm{M}}: 1.40 ; 95 \%\right.$ CI: 0.73 to 2.66 ). Players in U18 had significantly lower rates of injury, compared to U16 and U14 (IRR U16 $_{2}$ 2.44; 95\% CI: 1.22 to 4.87 ; IRR $\mathrm{U}_{14}$ : 3.58 ; $95 \% \mathrm{CI}: 1.60$ to 8.02 ).

Conclusion Players in U18 had the lowest injury rates. More research is needed to elucidate why younger age groups reported more injuries and develop volleyball specific injury and concussion prevention strategies.

\section{PERCEIVING, REPORTING AND MANAGING AN INJURY - PERSPECTIVES FROM NATIONAL TEAM FOOTBALL PLAYERS, COACHES AND HEALTH PROFESSIONALS}

1,2Sandro Vella, ${ }^{3}$ Caroline Bolling, ${ }^{3}$ Evert Verhagen, ${ }^{1}$ Isabel Sarah Moore. ${ }^{1}$ Cardiff School of Sport and Health Sciences, Cardiff Metropolitan University, Cardiff, UK; ${ }^{2}$ Malta Football Association, Millennium Stand, National Stadium, Ta Qali, Malta; ${ }^{3}$ Amsterdam Collaboration for Health and Safety in Sports, Department of Public and Occupational Health, Amsterdam Public Health Research Institute, VU University Medical Center, Amsterdam, Netherlands

\subsection{6/bjsports-2021-IOC.209}

Background Injury perceptions and related risk-mitigating interventions are context-dependent. Despite this, most injury surveillance systems guided by established consensus statements are not context-specific as they do not integrate stakeholders' perspectives. Accordingly, to address the injury problem in context, injury surveillance systems need to be aligned with the stakeholders' injury perceptions and practices related to its reporting and management.

Objective To explore how Maltese national team football players, coaches, and health professionals (stakeholders) perceive a football-related injury and how their context influences their perceptions and behaviours towards reporting and managing a football injury.

Design A qualitative exploratory design was used. 